\title{
Prescription pattern of urate-lowering therapy in Korean gout patients: data from the national health claims database
}

\author{
Ji-Won Kim ${ }^{1}$, Sang Gyu Kwak² ${ }^{2}$ and Sung-Hoon Park ${ }^{1}$
}

\author{
${ }^{1}$ Division of Rheumatology, De- \\ partment of Internal Medicine, \\ ${ }^{2}$ Department of Medical Statistics, \\ Catholic University of Daegu School \\ of Medicine, Daegu, Korea
}

Received: December 26, 2016 Accepted: January 17, 2017

\section{Correspondence to}

Sung-Hoon Park, M.D.

Division of Rheumatology,

Department of Internal

Medicine, Catholic University of Daegu School of Medicine, 33 Duryugongwon-ro 17-gil, Namgu, Daegu 42472, Korea

Tel: +82-53-650-4289

Fax: +82-53-629-8248

E-mail:youriig9@cu.ac.kr
Use of urate-lowering therapy (ULT) is indicated in gout patients with recurrent acute gout attacks ( $\geq 2 /$ year), tophi, chronic kidney disease stage $\geq 2$, and/or past urolithiasis [1]. Appropriate use of ULT decreased not only the risk of gout flares, but also renal and cardiovascular events during the follow-up period [2]. Previous reports have investigated that ULT was prescribed in 23\% (Taiwan), $38 \%$ (UK), and $42 \%$ (Sweden) of the gout population [3-5]. Xanthine oxidase inhibitors were the most common ULT prescribed in Sweden, whereas the uricosuric agents were the most common ULT prescribed in Taiwan. However, data on the prescription pattern of ULT are insufficient in Korea.

The aim of this study was to describe the prescription pattern of ULT in the South Korean gout population using the national health claims database 2007 to 2015 . Patients who had at least one health claim for gout (diagnostic code: M10) as a primary or secondary diagnosis from January 1,2007 , to December 31, 2015, were included in the present study. The proportion of patients who were prescribed ULT (allopurinol, benzbromarone, or febuxostat) among the total gout patients was calculated for each year from 2007 to 2015. For the patients who were prescribed ULT, the last prescribed drug for ULT in each year was investigated. In addition, we discovered the proportion of patients who were prescribed ULT each year during a 5-year period, without considering a refill gap.

Number and percentage of patients prescribed ULT and subsets of allopurinol, benzbromarone, and febuxostat were shown in Table 1. The total number of gout patients increased from 169,796 in 2007 to 383,471 in 2015 (annual prevalence: $0.35 \%$ in 2007 to $0.76 \%$ in 2015). ULT was prescribed in more than $80 \%$ of the gout population. However, the proportion of ULT prescription decreased in contrast to the increasing trend in prevalence of gout. Allopurinol was the most commonly used drug for ULT. The rate of allopurinol prescription steadily decreased from $96 \%$ in 2007 to $82 \%$ in 2015. Benzbromarone was used in 3\% to $4 \%$ of the ULT prescription. After febuxostat was approved in Korea, the use of febuxostat increased to $14 \%$ in 2015. Among gout patients who were prescribed ULT, only $33 \%$ to $38 \%$ of the patients were continuously prescribed ULT throughout a 5-year period. The proportion of patients who were continuously prescribed ULT was $33 \%$ in 2007 to 2012 period, which slightly increased to $38 \%$ in 2010 to 2015 period.

In contrast to studies conducted in 
Table 1. Gout patients who were prescribed ULT and proportion of allopurinol, benzbromarone, and febuxostat prescription

\begin{tabular}{lccccc}
\hline Year & Total gout & ULT & Allopurinol & Benzbromarone & Febuxostat \\
\hline 2007 & 169,796 & $146,261(86.1)$ & $140,887(96.3)$ & $5,374(3.7)$ & 0 \\
2008 & 189,231 & $161,674(85.4)$ & $155,303(96.1)$ & $6,371(3.9)$ & 0 \\
2009 & 208,247 & $178,360(85.6)$ & $170,662(95.7)$ & $7,698(4.3)$ & 0 \\
2010 & 233,397 & $198,750(85.2)$ & $189,862(95.5)$ & $8,888(4.5)$ & 0 \\
2011 & 253,975 & $213,299(84.0)$ & $203,930(95.6)$ & $9,369(4.4)$ & 0 \\
2012 & 285,838 & $237,238(83.0)$ & $213,260(89.9)$ & $10,062(4.2)$ & $13,916(5.9)$ \\
2013 & 321,419 & $265,219(82.5)$ & $221,623(83.6)$ & $10,335(3.9)$ & $33,261(12.5)$ \\
2014 & 346,685 & $285,466(82.3)$ & $236,106(82.7)$ & $10,809(3.8)$ & $38,551(13.5)$ \\
2015 & 383,471 & $313,098(81.6)$ & $257,912(82.4)$ & $10,827(3.5)$ & $44,359(14.2)$ \\
\hline
\end{tabular}

Values are presented as number (\%).

ULT, urate-lowering therapy.

other countries [3-5], our study revealed high rate of ULT prescription among the gout population. We assumed that the reason for this was the relatively low cost of this treatment for patients in Korea. Koreans are easily accessible to the health care service as all the population is covered by the National Health Insurance system and the Medical Aid program. Nevertheless, the continuation of ULT was still low. Patient-related factors and physician-related factors might contribute to the low rate of ULT continuation.

In conclusion, more than $80 \%$ of gout patients in Korea are prescribed ULT. Allopurinol is the most common ULT prescribed, but the use of febuxostat has increased in recent years. Among the patients who are prescribed ULT, only one-thirds are maintained with ULT during a 5-year period.

\section{Conflict of interest}

No potential conflict of interest relevant to this article was reported.

\section{Acknowledgments}

This work was supported by a grant from the Research Institute of Medical Science, Catholic University of Dae$\mathrm{gu}(2016)$.

\section{REFERENCES}

1. Khanna D, Fitzgerald JD, Khanna PP, et al. 2012 American College of Rheumatology guidelines for management of gout. Part 1: systematic nonpharmacologic and pharmacologic therapeutic approaches to hyperuricemia. Arthritis Care Res (Hoboken) 2012;64:1431-1446.

2. Goicoechea M, Garcia de Vinuesa S, Verdalles U, et al. Allopurinol and progression of CKD and cardiovascular events: long-term follow-up of a randomized clinical trial. Am J Kidney Dis 2015;65:543-549.

3. Kuo CF, Grainge MJ, See LC, et al. Epidemiology and management of gout in Taiwan: a nationwide population study. Arthritis Res Ther 2015;17:13.

4. Kuo CF, Grainge MJ, Mallen C, Zhang W, Doherty M. Rising burden of gout in the UK but continuing suboptimal management: a nationwide population study. Ann Rheum Dis 2015;74:661-667.

5. Dehlin M, Drivelegka P, Sigurdardottir V, Svard A, Jacobsson LT. Incidence and prevalence of gout in Western Sweden. Arthritis Res Ther 2016;18:164. 